livraisons

d'Histoire

de l'Architecture

\section{Livraisons de l'histoire de l'architecture}

24 | 2012

Le phare et l'architecte

\title{
André Pavlovsky et les feux de Saint-Jean-de-Luz, la modernité aux accents néo-basques
}

André Pavlovsky and the Harbor Lights of Saint-Jean-de-Luz, Modernity with Neo-Basque Accents

André Pavlovsky und die Leuchtfeuer in Saint-Jean-de-Luz. Modernität mit NeoBaskischen Akzenten

\section{Fabienne Doulat}

\section{OpenEdition}

\section{Journals}

Édition électronique

URL : http://journals.openedition.org//ha/97

DOI : $10.4000 /$ lha. 97

ISSN : 1960-5994

Éditeur

Association Livraisons d'histoire de l'architecture - LHA

Édition imprimée

Date de publication : 19 décembre 2012

Pagination : 107-124

ISSN : 1627-4970

Référence électronique

Fabienne Doulat, «André Pavlovsky et les feux de Saint-Jean-de-Luz, la modernité aux accents néobasques », Livraisons de l'histoire de l'architecture [En ligne], 24 | 2012, mis en ligne le 16 avril 2015, consulté le 01 mai 2019. URL : http://journals.openedition.org//ha/97 ; DOI : 10.4000//ha.97

Ce document a été généré automatiquement le 1 mai 2019.

Tous droits réservés à l'Association LHA 


\section{André Pavlovsky et les feux de Saint- Jean-de-Luz, la modernité aux accents néo-basques}

André Pavlovsky and the Harbor Lights of Saint-Jean-de-Luz, Modernity with

Neo-Basque Accents

André Pavlovsky und die Leuchtfeuer in Saint-Jean-de-Luz. Modernität mit NeoBaskischen Akzenten

Fabienne Doulat

En 1937, s'achève la construction des feux verts de Saint-Jean-de-Luz, monuments devenus emblématiques de la côte basque ${ }^{1}$. Ces constructions se démarquent des autres édifices de ce type non seulement par leur conception due à un architecte, André Pavlovsky (1891-1961)², et non à un ingénieur du service des Phares et balises comme il était alors d'usage, mais surtout par leur caractère régional marqué. C'est pourquoi il convient d'étudier les circonstances de cette édification atypique et d'envisager la façon dont elle s'insère dans la production d'un architecte dont les débuts sont empreints de néo-régionalisme avant de s'en détacher progressivement et d'affirmer un goût véritable pour la modernité.

\section{La construction des feux}

2 L'érection des feux de Saint-Jean-de-Luz est l'aboutissement d'un long travail de réflexion à la fois technique portant sur la façon dont les feux doivent être modifiés, mais aussi esthétique sur la conception de ces nouveaux édifices. 


\section{La nécessaire révision de l'éclairage de la baie de Saint-Jean}

3 Au début des années 1930 la dangerosité de la baie de Saint-Jean-de-Luz et les accidents de navigation qui s'y produisent régulièrement, amènent les autorités locales à demander au ministère des Travaux publics de rapides modifications de l'ensemble du système d'éclairage et de balisage de la zone. Celui-ci se compose à cette époque de plusieurs éléments distincts : les deux feux d'alignement rouges situés sur les hauteurs de la colline de Sainte-Barbe au Nord-Est de la baie, les deux feux d'alignement verts de Saint-Jean-deLuz et le feu fixe du port de Socoa à Ciboure (ill. 1).

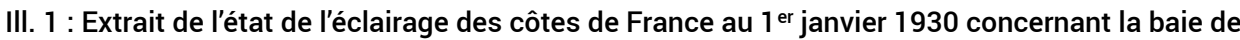
Saint-Jean-de-Luz, détail

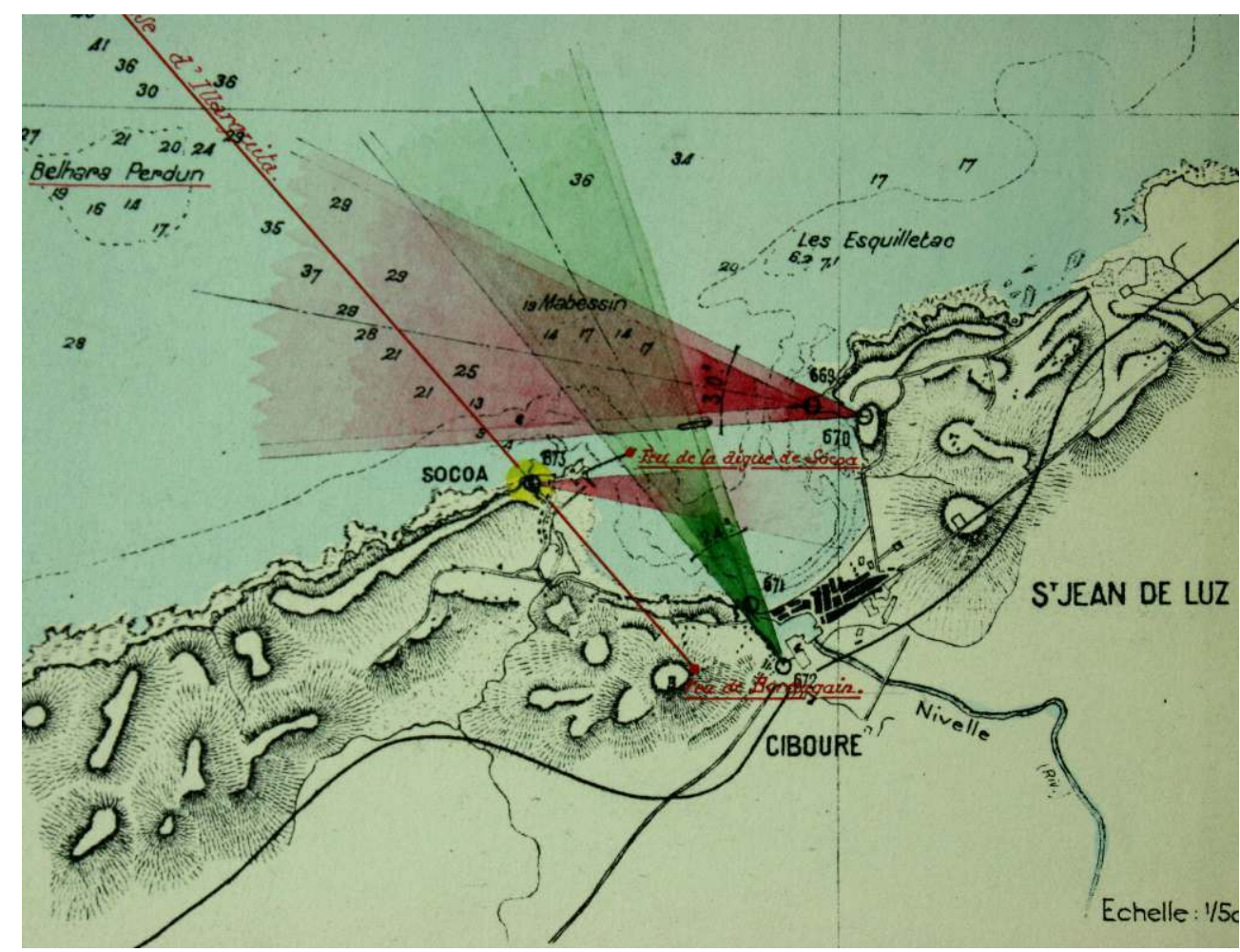

Carte imprimée avec ajouts à l'encre et au lavis, Arch. nat., F ${ }^{14} 20202$

Cl. O. Liardet.

4 Ces feux, alimentés au pétrole, ne présentent qu'une portée relativement faible. Les feux verts d'alignement, destinés à guider l'entrée en rade de Saint-Jean-de-Luz, sont considérés comme l'une des priorités du nouveau dispositif. Depuis 1870, le feu aval, placé sur la jetée de Saint-Jean-de-Luz sur la rive droite de l'embouchure de la Nivelle, est protégé par une cabane en tôle de deux mètres de hauteur. Le feu amont, dit aussi feu de Ciboure, est situé à 410 mètres du précédent, en bordure de la route nationale $\mathrm{n}^{\circ} 10$. Un feu provisoire fut établi en 1870 sur un échafaudage en bois avant d'être remplacé en novembre 1872 par une tour en maçonnerie (ill. 2). 
III. 2 : Élévation de la tour initiale du feu amont montrant la façade avec la porte d'entrée

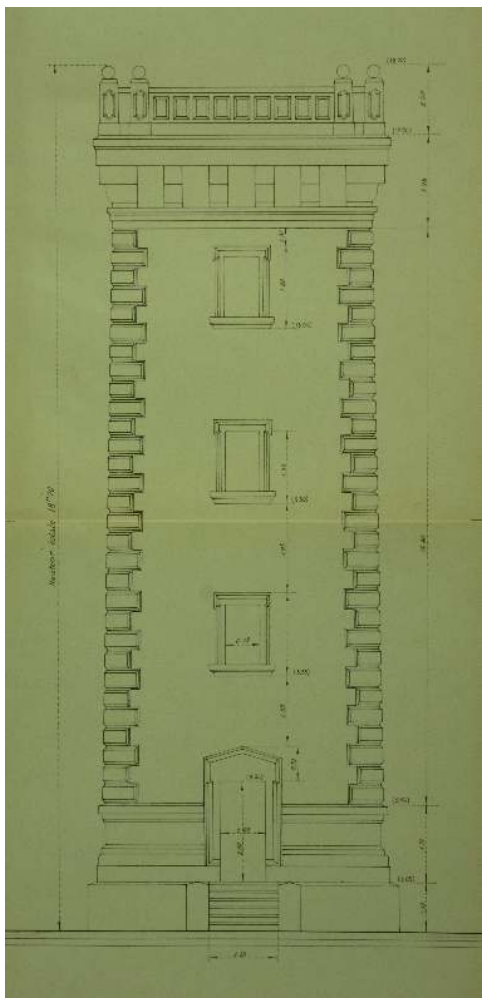

Dessin dressé par l'ingénieur ordinaire, 14 décembre 1935, tirage, Arch. nat., F¹4 20202

Cl. O. Liardet

5 L'édifice adopte un plan carré, dont l'aspect massif est accentué par un chaînage d'angle en bossage et une terrasse sommitale supportée par des consoles. En septembre 1912, ces feux furent modifiés afin d'accueillir un éclairage à incandescence alimenté par le gaz de houille.

Le préfet réunit une commission nautique le 22 septembre 1932, composée de responsables locaux de la navigation et de M. Lesbre, l'ingénieur des ponts et chaussée, afin d'étudier la question de l'éclairage et du balisage de la baie. Ces experts mettent en évidence le problème de visibilité des feux occasionné par l'éclairage des habitations et des voies publiques environnantes ainsi que par la digue de Socoa en ce qui concerne le feu aval. En s'appuyant sur ce travail, le service départemental des Phares et balises de l'arrondissement de Bayonne préconise le renforcement de la puissance des feux grâce à leur électrification et à l'exhaussement du plan focal, de onze à douze mètres en ce qui concerne le feu aval et d'une dizaine de mètres pour le feu amont, afin d'accroître leur portée et de les distinguer des autres lumières de la ville ${ }^{3}$. Ces propositions sont approuvées par André de Rouville (1882-1979), directeur des Phares et balises, dans sa lettre du 21 janvier 1935 et confirmées par une décision ministérielle le 15 avril suivant ${ }^{4}$.

\section{L'élaboration des projets}

7 L'ingénieur ordinaire présente le projet qu'il a dressé pour les feux, le 14 décembre 1935. En ce qui concerne le feu amont, l'idée, évoquée initialement, d'une tour métallique accolée à la construction est abandonnée pour des raisons esthétiques. L'ingénieure 
souligne d'ailleurs que «la tour s'encadre dans un paysage qu'il convient de ne pas déparer $»^{5}$. De même, la proposition d'augmenter la hauteur du bâtiment par une tourelle d'angle faite par de Rouville est rejetée par le Service départemental qui estime que « l'aspect de la tour actuelle se trouve alourdi de façon disgracieuse $»^{6}$ par cet ajout. Le rapport retient donc le principe d'une surélévation de la tour, tout en soulignant que le projet est complexe en raison de la hauteur du bâtiment demandée et qui revient pratiquement à doubler les proportions de l'édifice existant ${ }^{7}$. En outre, les murs épais et surtout l'implantation des fondations en terrain d'alluvions ne permettent pas une surélévation supérieure à huit mètres.

L'ingénieur expose dans son rapport deux solutions pour l'exhaussement de l'édifice. La première, élaborée par le Service départemental, consiste en "l'enlèvement du couronnement en pierre de taille, le renforcement des fondations et du socle de la tour, la surélévation de la tour et enfin la mise en place du couronnement $»^{8}$. La reprise des fondations qui représente un travail important est rendue nécessaire par le poids que la surélévation rajoute à la construction. Les ingénieurs estiment ces travaux à 200.000 francs. La deuxième proposition se fonde sur l'esquisse fournie par l'architecte départemental André Pavlovsky, sollicité à la demande du Service départemental (ill. 3) ${ }^{9}$

III. 3 : Photographie de l'esquisse de la tour amont projetée par Pavlovsky

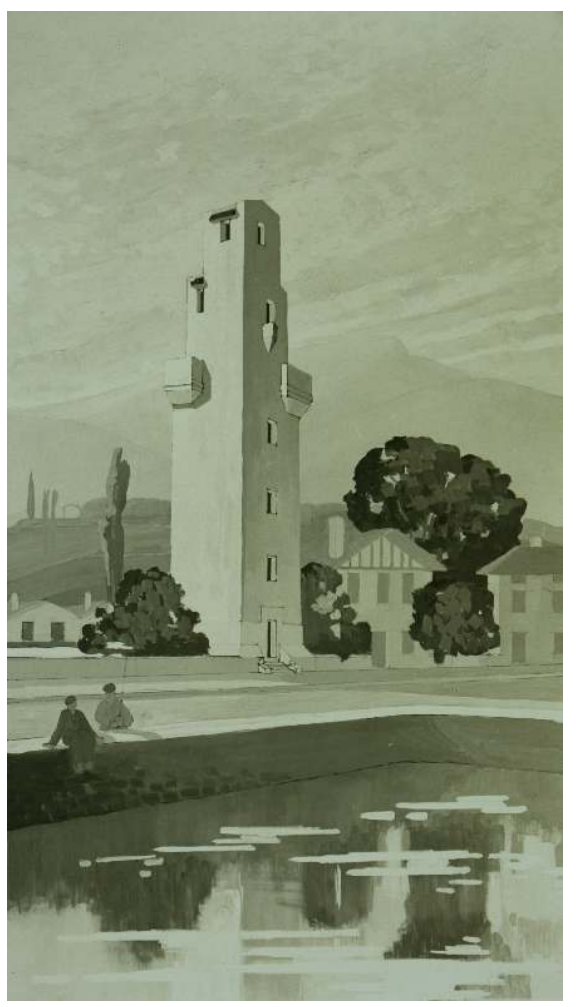

14 décembre 1935, Arch. nat., F ${ }^{14} 20202$

Cl. O. Liardet

9 Le projet prévoit que «pour ne pas surcharger exagérément les fondations de la tour actuelle [...] la surélévation serait constituée par une ossature et des parois en béton armé $»^{10}$. La partie ajoutée correspond à deux niveaux supplémentaires qui accueillent le système d'optique tandis que le reste de l'édifice est destiné au logement du gardien ${ }^{11}$ .Cette solution, évaluée à 70.000 francs, s'avère moins coûteuse que la première car elle 
permet d'éviter les travaux importants de renforcement des fondations. Le Service départemental affirme clairement sa préférence pour cette solution, à la fois plus avantageuse d'un point de vue financier et plus satisfaisante sur le plan esthétique (ill. 4).

III. 4 : Élévation du feu amont projeté $2^{\mathrm{e}}$ solution, côté large

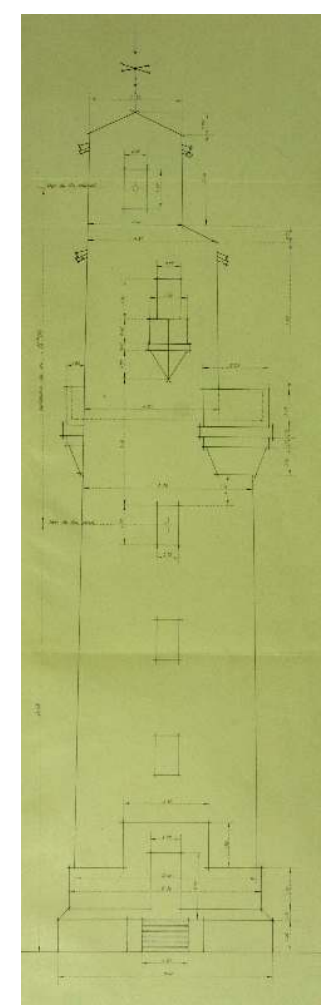

14 décembre 1935, tirage, Arch. nat., F ${ }^{14} 20202$

Cl. O. Liardet

10 Le rapport de l'ingénieur envisage également la question de l'établissement du nouveau feu aval, situé à l'emplacement de celui existant pour respecter l'alignement avec le feu amont. La solution d'un pylône métallique à treillis est rapidement écartée par l'ingénieur car elle ne permet pas une visibilité suffisante et engendrerait des coûts de construction et d'entretien importants. Le service se prononce donc en faveur de l'érection d'une tour en béton armé dans le même style que la future tour amont afin de respecter les exigences esthétiques adoptées pour cette dernière, d'autant que ce feu est particulièrement visible (ill. 5). 


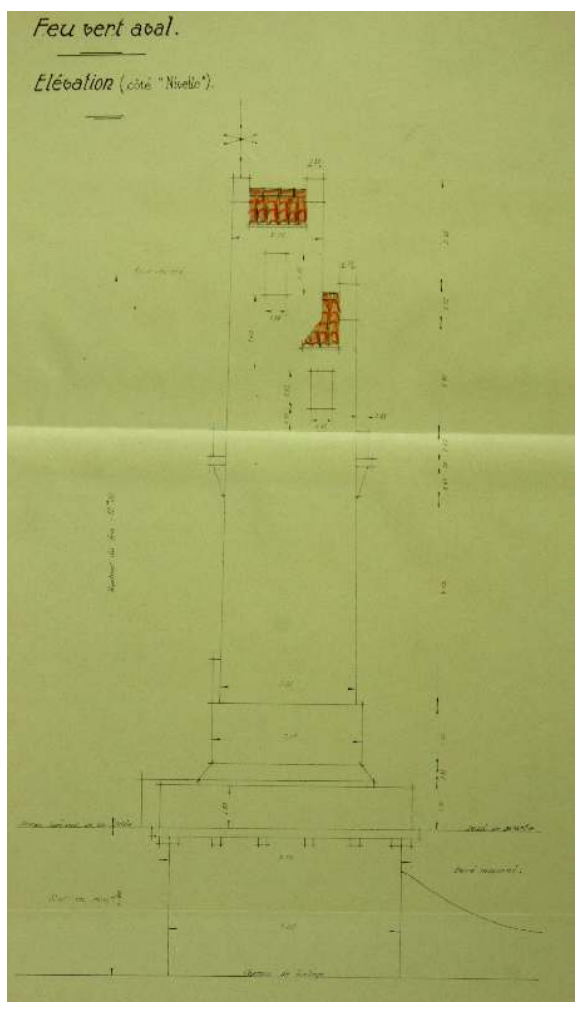

14 décembre 1935, tirage colorié, Arch. nat., F ${ }^{14} 20202$

Cl. O. Liardet.

Les travaux apparaissent complexes car étant donné la position retenue pour l'édifice, les fondations doivent empiéter sur la jetée et sur le "seuil de garantie», dont les constructions présentent des signes de fragilité. Il n'est ainsi pas envisageable d'asseoir le nouveau feu sur ces maçonneries, ce qui nécessite d'établir des fondations profondes et indépendantes pour le nouvel édifice. Ce projet de reconstruction du feu aval est évalué à 110.000 francs.

12 Les propositions présentées par l'ingénieur sont approuvées par le ministre des Travaux publics le 9 mars 1936 qui arrête :

« une surélévation, un renforcement et l'adjonction d'un rythme pour les deux feux de l'alignement de Ciboure qui seront respectivement placés à $27 \mathrm{~m}$ et à $17 \mathrm{~m} 50 \mathrm{au}-$ dessus du niveau des hautes mers. Ils auront une visibilité moyenne de 20 milles et présenteront synchroniquement deux occultations groupées toutes les 6 secondes en lumière verte $»^{12}$.

13 Étant donné le rôle majeur de ces feux dans la navigation, leur électrification nécessite un éclairage de secours pour éviter toute interruption en cas de coupure de courant. Cette alimentation doit se faire au gaz grâce à une canalisation située à proximité. Le projet d'exécution des travaux est dressé par le Service départemental en collaboration avec Pavlovsky pour le mois de novembre suivant. Les travaux préparatoires à l'exhaussement du feu amont consistent en la dépose et la descente de la balustrade en pierre de la tour maçonnée, puis l'abattage et le recoupement de la saillie de la corniche dans les parties supprimées. La surélévation doit être réalisée en béton armé en ce qui concerne la structure et l'intérieur tandis que les pignons sont en briques creuses. Le plancher haut du troisième étage, qui forme la toiture, est partiellement détruit pour laisser le passage à 
l'escalier. L'intérieur de la partie exhaussée doit recevoir un enduit de chaux hydraulique à deux couches. À l'extérieur, la partie existante subit le recoupement des saillies du bossage d'angle et des chambranles, le piquetage du soubassement avant que l'ensemble de l'édifice ne reçoive un enduit en mortier de ciment peint. Les travaux projetés pour le feu aval présentent les mêmes caractéristiques que ceux du feu amont en ce qui concerne la construction proprement dite. Ils nécessitent toutefois la démolition au préalable de la maçonnerie de la jetée par petites parties pour permettre l'établissement des fondations. Celles-ci consistent en douze pieux armés d'environ douze mètres de long et 0,42 mètres de diamètre, destinés à accueillir un hourdis général devant supporter l'ensemble de la construction. Les travaux du feu aval ne doivent commencer qu'après la réalisation de l'exhaussement du feu amont afin d'assurer la visibilité des feux pendant toute la durée des travaux.

14 À la suite de l'appel d'offre l'entrepreneur retenu, A. Margeridon, s'engage à réaliser les travaux pour la somme de 155.200 francs $^{13}$. Les travaux de fondation, qui font l'objet d'un marché spécial, sont confiés pour le prix forfaitaire de 60.000 francs à une entreprise spécialisée, la société Les Fondations Modernes, qui exploite le système Frotté. À ces dépenses s'ajoutent celle de 64.800 francs pour les travaux imprévus, l'installation de l'éclairage normal et de secours ainsi que la surveillance des travaux, ce qui porte le total à 280.000 francs. Le projet d'exécution de la transformation des feux reçoit l'approbation ministérielle le 28 décembre 1936 puis l'autorisation de dépense ${ }^{14}$. Par la suite, une demande de crédit supplémentaire est présentée le 18 octobre 1937 pour l'électrification des feux, décidée le 6 août précédent. L'entreprise Dussouy est désignée après appel d'offre pour effectuer la pose du câble de liaison des deux feux ainsi que le branchement du feu amont sur le secteur local de distribution d'après un devis de 45.000 francs. Le Service départemental demande à la même entreprise de s'occuper des travaux d'éclairage des deux édifices afin que les travaux puissent être réalisés en même temps, ce qui correspond à une nouvelle dépense de 3.800 francs. Avec les autres frais généraux le crédit nécessaire pour la surélévation et le renforcement par électrification des feux s'élèvent à 302.000 francs au lieu des 269.000 francs déjà alloués. Les travaux s'achèvent à la fin de l'année 1937 puis en décembre 1938, les feux sont électrifiés et une lentille de Fresnel est installée ${ }^{15}$.

\section{Le rôle joué par l'architecte}

15 La conception des feux de Saint-Jean-de-Luz par un architecte constitue une particularité qu'il convient de souligner car les phares sont traditionnellement élaborés par les ingénieurs du service des Phares et balises. Le partage des rôles est d'ailleurs clairement défini puisqu'il revient aux Services départementaux d'établir les ouvrages proprement dits, tandis que le choix et la fourniture de l'appareillage concernant l'électrification des feux dépend du Service central. La situation de Saint-Jean-de-Luz est exceptionnelle pour la première moitié du $\mathrm{XX}^{\mathrm{e}}$ siècle et l'on ne peut guère citer comme autre exemple que le feu Saint-Pol bâti à Dunkerque par l'architecte Gustave Umbdenstock (1866-1940) en 1937-38, alors que la conception des phares par des architectes deviendra courante après la guerre. Comme nous l'avons signalé, il revient au Service bayonnais de prendre l'initiative de faire appel à Pavlovsky pour l'aider, non seulement dans le dessin des feux, mais aussi l'ensemble de la mise au point du projet d'exécution. L'ingénieur de l'arrondissement justifie cette démarche par la surcharge de travail, mais cela paraît une 
explication un peu courte pour une décision aussi originale. En l'absence de documentation sur le contexte de cette collaboration, il serait hasardeux d'en tirer des conclusions, mais on peut remarquer que l'ingénieur soutient le projet de Pavlovsky avec fermeté tout au long du processus. Le service s'est néanmoins réservé la partie la plus technique du travail, à savoir les fondations du feu aval représentant une difficulté particulière dans ce cas. L'intervention de l'architecte ne semble toutefois pas être bien perçue par la direction parisienne. Sans critiquer le recours à une personnalité extérieure, le directeur du service des Phares et balises rappelle que cela ne doit pas « entraîner une substitution des rôles, en conférant à M. Pavlovsky [...] employé en fait à titre auxiliaire et momentané, la qualité de maître de l'ouvrage qui appartient en propre à l'Ingénieur représentant de l'Administration ${ }^{16}$. Il demande donc que toutes les références à l'architecte soient enlevées des documents officiels pour être remplacées par le terme l'« ingénieur ou son préposé » et que soient également supprimées les évocations des honoraires. On constate d'ailleurs que dans le projet d'exécution du 8 décembre 1936 les références à l'architecte sont en effet systématiquement biffées. La direction estime en outre que « la rémunération de l'architecte doit être envisagée comme un émolument ou un salaire (à forfait, au mois ou à la journée) $»^{17}$ qu'il revient à l'ingénieur d'intégrer au budget. L'existence d'honoraires pourrait en effet créer pour l'architecte une responsabilité décennale qui doit revenir de fait à l'administration.

Cette situation explique sans doute l'absence, mise à part l'esquisse originale, de documents élaborés par l'architecte. Le défaut d'archives personnelles ou de l'agence de Pavlovsky nous prive donc d'informations capitales sur la démarche de l'architecte dans l'élaboration de ce projet particulier et sur son implication dans le chantier. Il conserve cependant une certaine autorité sur son travail puisque l'ingénieur Lesbre le consulte sur les modifications suggérées par le directeur du service des Phares et balises après son inspection et qu'il obtient en général gain de cause.

\section{Les feux de Saint-Jean-de-Luz, une synthèse aboutie}

17 Les bâtiments des feux de Saint-Jean-de-Luz présentent un grand intérêt non seulement en raison de leur esthétique originale mais aussi par la place qu'ils occupent dans la carrière de leur concepteur et, plus largement, dans l'évolution de l'architecture propre au Pays basque.

\section{Une esthétique originale}

Les feux proposés par Pavlovsky, qui développent les mêmes principes de composition, ont une esthétique très différente de l'architecture traditionnelle des phares. La structure carrée permet le dégagement de murs pignons et de toits à deux pans recouverts de tuiles canal en débord sur les façades latérales. Les faces sud des deux édifices complexifient ce principe puisque elles se composent de deux murs pignons superposés, dont le plus haut est situé en retrait (ill. 6). 
III. 6 : Vue de la façade nord du feu amont avec la bande de couleur verte et l'ancienne porte transformée en fenêtre

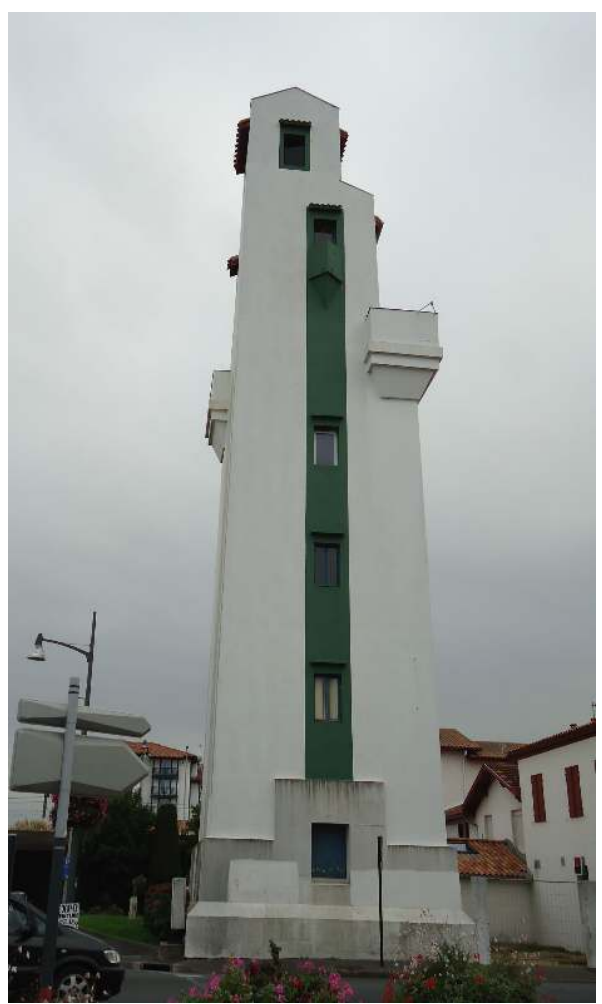

Cl. Pascale Diharce

Ce jeu de décrochement, qui accentue l'effilement des édifices, est particulièrement sensible $\mathrm{du}$ fait qu'aucune face n'est semblable aux autres. La verticalité est une caractéristique du travail de l'architecte et se retrouve dans d'autres de ses œuvres. Ainsi l'allure générale des feux doit beaucoup aux trois phares dessinés pour le monument en mémoire de Christophe Colomb, avec lesquels ils partagent le même élancement et le principe d'étagement. Cette verticalité est aussi très présente dans les villas réalisées par l'architecte au travers de la mise en avant des souches de cheminée, à la fois ornement et élément de composition et dont certaines se rapprochent des feux de Saint-Jean-de-Luz.

Les feux affichent une grande sobriété et sont dépouillés d'ornements, comme souvent dans l'architecture de Pavlovsky. L'architecte utilise seulement quelques éléments en relief qui, grâce au jeu d'ombre et de lumière qu'ils provoquent, créent un mouvement sur les grandes surfaces blanches des murs. Ainsi le feu amont, qui englobe dans le nouvel édifice la tour existante, transforme l'ancien garde-corps en encorbellement en une terrasse enveloppant partiellement le bâtiment (ill. 7). 
III. 7 : Vue de la partie supérieure du feu amont, la terrasse surmontée de la partie ajoutée par Pavlovsky

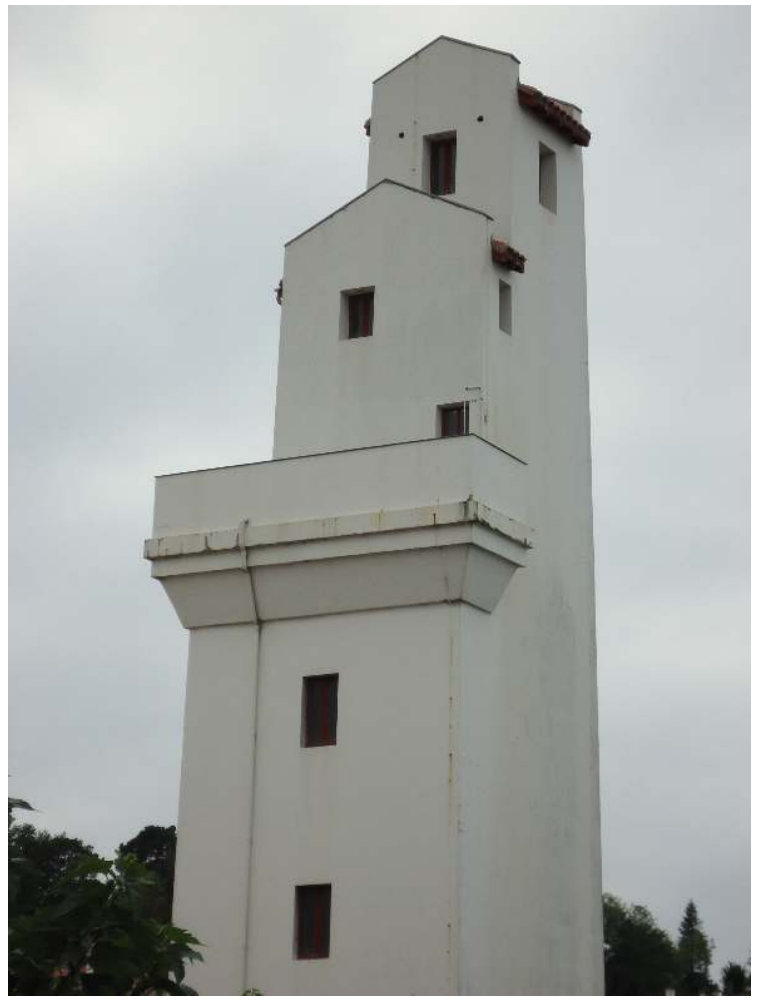

Cl. Pascale Diharce

21 Celle-ci permet de relier les façades les unes aux autres et d'animer la construction, selon un principe que l'architecte développe également dans certains balcons d'angle de ses villas. Cet élément apparaît d'ailleurs comme une volonté délibérée de l'artiste, ce qu'attestent les échanges entre l'ingénieur et le directeur du service des Phares et balises à propos de modifications à apporter au projet. Le directeur demande en effet le «rescindement du saillant sur la face Est de la terrasse du feu amont». Pavlovsky s'oppose à cette modification exposant qu'il a « intentionnellement [...] ménagé un relief coupant la face Est au droit du raccord entre l'ancienne tour et l'exhaussement $\aleph^{18}$. Il estime en effet que "ceci est essentiel pour l'aspect côté Saint-Jean-de-Luz (route nationale $\left.n^{0} 10\right) »^{19}$, d'autant qu'il s'agit de la façade la plus exposée aux regards. Même s'il n'est pas favorable à la saillie du balcon, le directeur cède devant les arguments de l'architecte. Les baies du deuxième étage au nord et au sud du feu aval ainsi que la troisième baie de la façade nord du feu amont sont agrémentées de balcons en étrave (ill. 8). 
III. 8 : Vue du sommet du feu aval et des détails décoratifs, côté sud

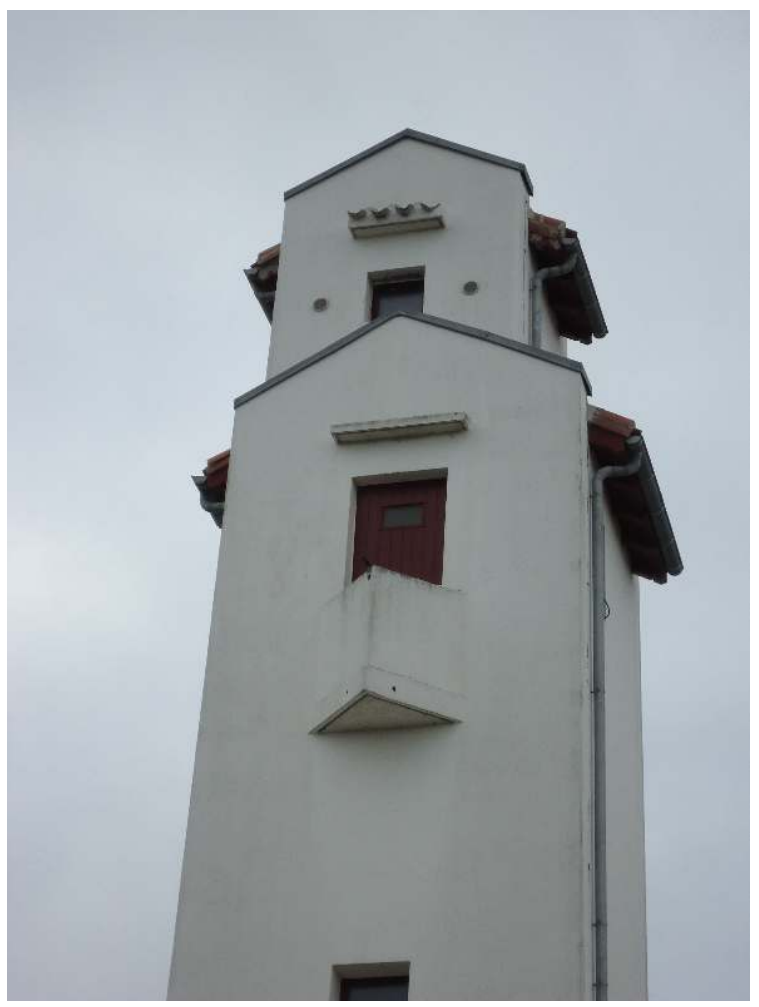

Cl. Pascale Diharce

22 Cette évocation maritime évidente participe aussi à une sobre mise en relief des façades. De même de petits bandeaux moulurés ou de petits avant-toits en tuile au-dessus de certaines fenêtres animent les murs lisses par ailleurs. Pavlovsky met donc à profit tous les effets graphiques que peuvent apporter ces détails constructifs. Ces éléments décoratifs ne sont toutefois pas systématiques et accentuent l'impression de dissymétrie que provoquent déjà les étagements des constructions et la disposition des fenêtres (ill. 9). 


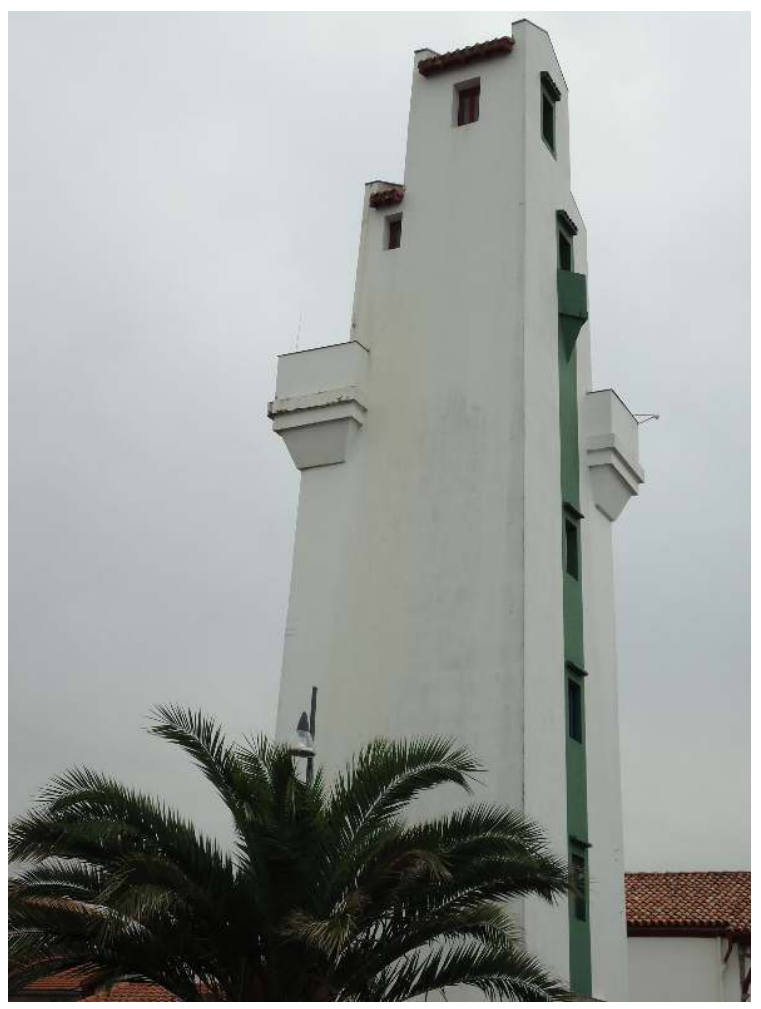

Cl. Pascale Diharce

Actuellement l'un des éléments décoratifs les plus évidents est apporté par les bandes verticales de couleur qui viennent souligner la superposition des ouvertures, de la porte d'entrée aux fenêtres, sur les façades Nord, c'est-à-dire côté océan, des deux édifices. À l'origine, ces bandes étaient peintes en rouge mais, en 1957, celle du feu amont devient verte, évoquant ainsi les couleurs du Pays basque. Ce qui apparaît comme un principe fondateur de ces constructions ne semble toutefois pas être voulu initialement par Pavlovsky. En effet, lors de la mise au point du projet, Rouville propose que soit peints en brun divers éléments de la face nord du feu aval. Par contre, l'architecte souhaite, s'il n'y a pas de raison liée à la navigation, que l'édifice soit laissé totalement blanc comme il l'a prévu. Cette couleur doit permettre de rendre l'édifice plus visible et de donner des effets de lumière. Le directeur se montre tenace sur cette question, estimant que la visibilité de la tour serait améliorée si certaines parties de la façade étaient colorées en brun «Van Dyck ». Il propose donc à l'ingénieur de peindre dans un premier temps la totalité de la façade en blanc puis de «faire badigeonner de vieilles toiles ou de vieux papiers dans la couleur prévue et les faire présenter, de manière à voir si, à la fois, l'aspect du bâtiment n'y perd rien et la visibilité n'y gagne pas quelque chose ${ }^{20}$. L'expérience semble avoir été concluante car l'idée a été en définitive retenue et même généralisée au deuxième feu. 


\section{L'intégration à un style régional ou la manifestation d'une démarche spécifique} relation, commencée dès l'enfance lors des séjours estivaux effectués avec sa famille. C'est donc très naturellement qu'après ses études à l'École des beaux-arts de Paris, dont il est diplômé en 1920, et ses premiers chantiers menés dans le Nord jusqu'en 1924 avec Louis Quételart (1888-1950), il décide de s'installer à Saint-Jean-de-Luz, où il fondera son foyer. La région poursuit à cette époque son spectaculaire développement, amorcé dès le Second Empire, et offre à un jeune architecte de nombreuses opportunités de travail auprès d'une cliente fortunée et cosmopolite. Cet essor remarquable fait de cet endroit, comme d'autres stations balnéaires, le théâtre de riches expérimentations architecturales. Si les styles éclectique ou historiciste y sont au XIXe siècle privilégiés, en particulier à Biarritz, les architectes développent à partir de la fin des années 1890 un style néo-basque, qui représente l'un des courants forts et précoces du mouvement régionaliste architectural alors en pleine ascension ${ }^{21}$. La référence retenue est la ferme labourdine dont les pans de bois, les toits à long pans, les encorbellements et les épais murs de refend en saillie alimentent les créations. Durant l'entre-deux-guerres, alors que le succès de la région ne se dément pas, les chantiers de constructions particulières mais aussi d'équipements collectifs se multiplient. Néanmoins dans les années 1920-1930, le style néo-basque prend du recul par rapport à ses modèles originaux, devenant plus conceptuel et théorique, sous l'impulsion en particulier d'Henri Godbarge $(1872-1946)^{22}$ et de Benjamin Gomez $(1885-1959)^{23}$. Le modèle architectural basque permet parfaitement de surmonter la crise que connaît l'architecture balnéaire à cette époque car il est plus facilement conciliable que d'autres styles régionaux avec le retour à un certain classicisme qui caractérise cette période.

Grâce à sa connaissance approfondie de la région, Pavlovsky s'inscrit dans ce mouvement néo-basque. Il distille d'ailleurs des éléments caractéristiques de cette culture dès ses premiers chantiers même s'il est alors très influencé par le travail de Dom Bellot (1876-1944). Il recourt ainsi au répertoire des formes basques pour agrémenter avec parcimonie ses bâtiments de la reconstruction dans le Nord, comme à l'église de VieilleChapelle où l'on trouve le motif de la virgule ${ }^{24}$. Les premières réalisations qu'il produit dans les environs de Saint-Jean-de-Luz dans les années 1925-1926, de belles villas pour de riches commanditaires, adoptent un style néo-basque marqué. Il faut toutefois souligner que le travail de l'architecte se caractérise dès le début de sa carrière par une sobriété décorative et une absence de recherche du pittoresque qui le place déjà un peu à part du courant régionaliste. Dès la fin de 1926, il propose dans ces résidences une interprétation libre des principes de l'architecture du pays. Comme ses confrères, il saura également se renouveler en puisant son inspiration dans l'architecture des autres régions basques, puis en se tournant vers l'Espagne. Cette diversification des sources permet d'échapper au risque d'enlisement que fait courir le succès rencontré par le style néo-basque et se voit pratiquée aussi par William Marcel (1879-1971), notamment à la mairie d'Anglet, ou les frères Gomez dans de nombreuses villas. 
Sans renier le style néo-basque, l'artiste se montre particulièrement sensible au débat architectural de l'époque entre les régionalistes et les adeptes de la modernité, se tenant informé de l'actualité de ces derniers à travers les revues architecturales et les publications. Dès cette période, son attirance pour ce style est perceptible et il développe des projets plus modernes lorsqu'il en a la possibilité comme à l'hôtel Édouard VII édifié en 1927. L'année suivante, il franchit un pas supplémentaire vers le modernisme comme en témoigne le projet de trois tours qu'il présente au concours pour le phare monumental à la mémoire de Christophe Colomb en République Dominicaine. Les villas qu'il réalise à cette époque autour du golf de Chantaco, illustrent également l'évolution de son travail, adoptant des plans irréguliers inspirés par l'architecture moderne internationale tout en utilisant des références au vocabulaire basque. Selon le commanditaire ou le programme auquel il est confronté, il s'offre un retour à une architecture plus proche de la tradition. Il faut remarquer que les réalisations dans le style moderne sont assez peu nombreuses dans cette région même si le célèbre casino de Saint-Jean-de-Luz réalisé en 1928 par Rob Mallet-Stevens (1886-1945) en est un éclatant exemple. Pavlovsky utilise également quelques références à l'Art Déco dans ses réalisations, sans pour autant adopter ce style comme à pu le faire Joseph Hiriart (1888-1946) dans sa fameuse villa Leihorra à Ciboure en 1928 ou Alfred Laulhé (1879-1956) au nouveau casino de Biarritz en 1929. Pavlovsky illustre bien la démarche des architectes de l'entre-deux-guerres œuvrant au Pays basque qui, tout en partant d'un style régionaliste, développent leur propres esthétique, se tournant au gré des chantiers vers d'autres références. C'est ainsi que Maurice Culot, dans l'ouvrage qu'il a consacré à l'architecte, peut le définir comme « un moderniste qui refuse de faire table rase du passé mais qui, au contraire, recherche dans l'étude de l'architecture régionale les moyens d'une synthèse originale ${ }^{25}$.

Les feux de Saint-Jean-de-Luz témoignent de l'évolution de la conception architecturale de Pavlovsky et s'inscrivent dans le tournant plus moderne que prend l'architecte au début des années 1930, tout en illustrant son attachement à la tradition basque. Loin des éléments couramment utilisés dans le style néo-basque comme les détails décoratifs, la multiplication des ouvertures et le jeu polychromique entre le bois, la pierre et les enduits, Pavlovsky ne retient de l'architecture basque que ses éléments structurels. Les feux mettent ainsi en valeur le rôle central du mur pignon, les toits à deux pans débordants souvent asymétriques, la façade principale percée d'ouvertures tandis que les autres sont pratiquement aveugles, l'aspect massif et les formes rectilignes ainsi que les grands pans de murs laissés blancs qu'offre l'architecture basque. L'architecte résume d'ailleurs sa pensée à la fin de sa vie en déclarant qu'« il ne faut jamais copier servilement mais interpréter et ne respecter que l'idée générale des anciens, en un mot évoluer ${ }^{26}$. Il privilégie un travail de composition dont la décoration repose sur les effets plastiques de la construction qui confère leur évidente modernité aux édifices luziens. Pavlovsky atteste par là-même de l'indéniable adaptabilité de l'architecture basque aux principes modernes et il propose avec les feux de Saint-Jean-de-Luz une synthèse de ces deux courants stylistiques souvent opposés (ill. 10). 
III. 10 : Vue du feu aval, côtés nord et ouest, avec les fondations qui englobent la jetée

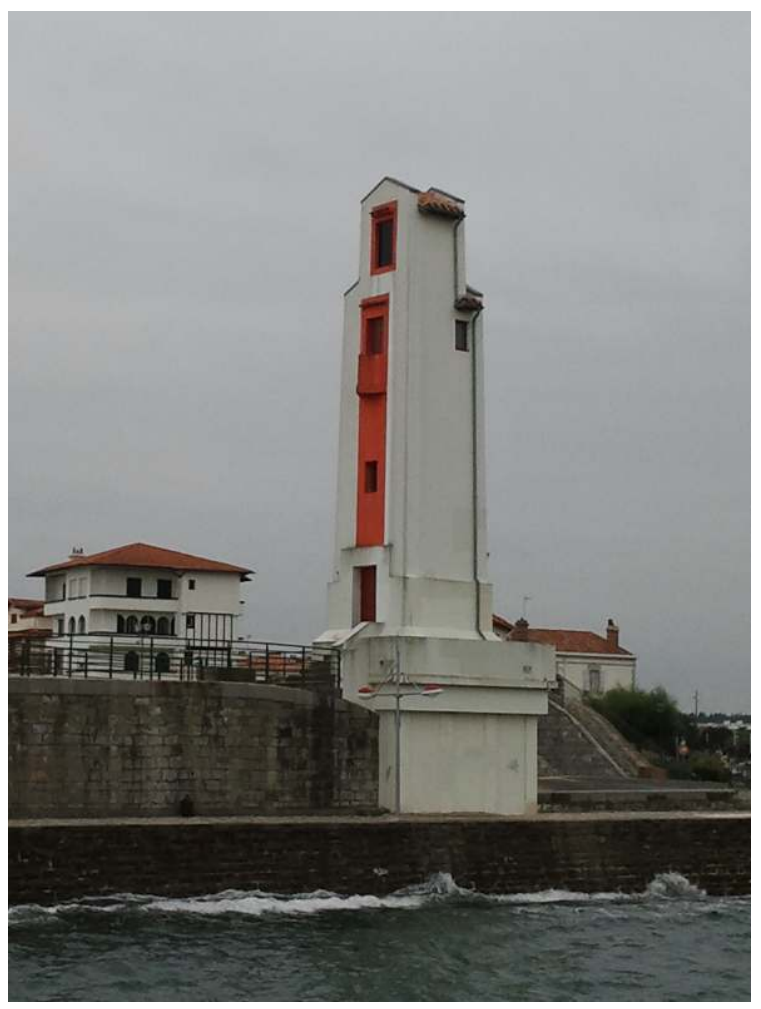

Cl. Pascale Diharce.

\section{NOTES}

1. Les deux feux sont inscrits à l'inventaire supplémentaire des monuments historiques par arrêté du 8 octobre 1993.

2. Sur cet architecte voir Maurice Culot et Jacques Pavlovsky, Architectures d'André Pavlovsky: la Côte basque des années trente, Paris, 1991, 207 p.

3. Arch. nat., $\mathrm{F}^{14} 20202,2^{\mathrm{e}}$ rapport de l'ingénieur, 27 décembre 1934.

4. Cité dans Arch. nat., $\mathrm{F}^{14}$ 20202, $2^{\mathrm{e}}$ rapport de l'ingénieur, 14 décembre 1935.

5. Arch. nat., $\mathrm{F}^{14}$ 20202, $2^{\mathrm{e}}$ rapport de l'ingénieur, 14 décembre 1935.

6. Arch. nat., $\mathrm{F}^{14} 20202,2^{\mathrm{e}}$ rapport de l'ingénieur, 14 décembre 1935.

7. La tour, de plan carré, compte 6,80 mètres de côté et 17,20 mètres de hauteur. Le foyer lumineux se trouve à 14 mètres.

8. Arch. nat., $\mathrm{F}^{14} 20202,2^{\mathrm{e}}$ rapport de l'ingénieur, 14 décembre 1935.

9. Pavlovsky est nommé architecte départemental des Basses-Pyrénées pour l'arrondissement de Bayonne en 1932 et assume cette fonction jusqu'à son décès.

10. Arch. nat., $\mathrm{F}^{14} 20202,2^{\mathrm{e}}$ rapport de l'ingénieur, 14 décembre 1935.

11. En 1959, un logement est construit au pied de la tour ce qui entraine la transformation de la porte d'entrée, côté nord, en simple fenêtre. 
12. Arch. nat., $\mathrm{F}^{14}$ 20202, rapport de l'ingénieur, 8 décembre 1936.

13. Arch. nat., $\mathrm{F}^{14}$ 20202. Le devis estimatif dressé par l'ingénieur prévoyait $94.475,69 \mathrm{f}$. pour le feu amont et $80.005,40 \mathrm{f}$. pour le feu aval, soit un montant total de $174.481,09 \mathrm{f}$.

14. Le crédit de 28000 francs est ouvert sur le chapitre $G$ (fond d'outillage) du budget du ministère des travaux publics le 23 décembre 1936.

15. 0,1875 mètre de distance focale synchronisée de deux occultations en six secondes. Le rythme des feux est modifié en 1986 avec un scintillement continu.

16. Arch. nat., $\mathrm{F}^{14} 20202$, lettre d'A. de Rouville, directeur du service des Phares et balises, à Blanchet, ingénieur en chef, 23 décembre 1936.

17. Arch. nat., $\mathrm{F}^{14} 20202$, lettre d'A. de Rouville, directeur, à Blanchet, ingénieur en chef, 23 décembre 1936.

18. Arch. nat., $\mathrm{F}^{14}$ 20202, lettre d'A. de Rouville, directeur, à Lesbre, ingénieur, 18 octobre 1937.

19. Arch. nat., $F^{14} 20202$, lettre d'A. de Rouville, directeur, à Lesbre, ingénieur, 18 octobre 1937.

20. Arch. nat., $\mathrm{F}^{14}$ 20202, lettre d'A. de Rouville, directeur, à Lesbre, ingénieur, 18 octobre 1937.

21. Sur la question du régionalisme voir notamment Jean-Claude Vigato, L'architecture régionaliste: France, 1890-1950, Paris, 1994, 390 p.; Monuments historiques, $\mathrm{n}^{\circ} 189$, sept.-oct. 1993, "le régionalisme ", et François Loyer et Bernard Toulier dir., Le régionalisme, architecture et identité, Paris, 2001, 279 p. Sur le néo-basque voir entre autre Jean-Claude Lasserre, «Le néo-basque (1920-1940)», Monuments historiques, n 147, oct.-nov. 1986, p.65-72; Geneviève Mesuret et Maurice Culot, Architectures de Biarritz et de la Côte basque, de la belle époque aux années trente, LiègeBruxelles, 1990, 142 p.; Vieilles Maisons Françaises, $\mathrm{n}^{\circ} 224$, sept. 2008, «Le Pays basque, architectures et identité ».

22. Claude Laroche, «Henri Godbarge. Le néo-basque prospectif », Monuments historiques, $\mathrm{n}^{\circ} 189$, sept.-oct. 1993, p. 74-78.

23. Jean Idiart, Claude Laroche et Évelyne Bacardartz, Louis et Benjamin Gomez: architectes à Bayonne 1905-1959, Bayonne, 2009, 159 p.

24. Richard Klein, «Pavlovsky et Quételart. Un savoureux mélange des genres », Monuments historiques, $\mathrm{n}^{\circ}$ 189, sept.-oct. 1993, p. 91-93.

25. M. Culot et J. Pavlovsky, Architectures d'André Pavlovsky, op. cit., p. 64.

26. André Pavlovsky, «Landes. Pays Basque. Béarn. De l’Océan aux Pyrénées », Plaisir de France. Styles régionaux: architecture, mobilier, décoration. Provence, Flandre, Artois, Picardie, Landes, PaysBasque, Béarn, Alsace, Bretagne, Paris, s. d. [1959], p.92 cité dans M. Culot et J. Pavlovsky, Architectures d'André Pavlovsky, op. cit., p. 56.

\section{RÉSUMÉS}

En 1937, s'achèvent les travaux des feux verts de Saint-Jean-de-Luz réalisés d'après le projet de l'architecte André Pavlovsky (1891-1961). Cette collaboration exceptionnelle entre un architecte et le service des Phares et balises, ne se fait d'ailleurs pas sans quelques tensions. L'exhaussement de la tour existante $\mathrm{du}$ feu amont et la construction du nouveau feu aval adoptent une architecture originale non seulement par rapport aux phares traditionnellement construits mais aussi dans le paysage basque. Ces deux constructions élancées se caractérisent par un jeu subtil de décrochement des volumes et une décoration très sobre, fondée sur quelques éléments de relief animant les façades blanches grâce à la lumière. Pavlovsky, dont le début d'activité est 
marqué par un style néo-basque épuré, se détache ici des références régionalistes pour proposer une œuvre beaucoup plus moderne, comme il aime à le faire quand il en a la possibilité à partir de la fin des années 1920. Les feux de Saint-Jean-de-Luz constituent donc une illustration de cette synthèse que l'architecte a voulu proposer entre une modernité assumée et la tradition basque à laquelle il reste attaché.

In 1937, work on the green lights of Saint-Jean-de-Luz (Pyrénées-Atlantiques) designed by André Pavlovsky (1891-1961) was completed. This collaboration between an architect and the Department of Lighthouses and Beacons was exceptional in its time and not without tension. The project consisted of heightening an existing lighthouse tower and constructing a second one, both adopting an original architectural expression distinct from both traditional lighthouse forms and local Basque construction. The two slender towers are characterized by a subtle uncoupling of volumes and very sober relief decorations that animate the white walls with light and shadow. Pavlovsky, whose early work is marked by a sleek, neo-Basque style, abandons regionalist references in his lighthouse designs to produce a much more modern aesthetic. The lighthouses of Saint-Jean-de-Luz thus exemplify the synthesis of modernity and Basque tradition that Pavlovsky endeavored to achieve in his mature work, beginning in the late 1920s.

Im Jahre 1937 endeten die Bauarbeiten der grünen Leuchtfeuer von Saint-Jean-de-Luz, die nach dem Projekt des Architekten André Pavlovsky errichtet wurden. Diese Zusammenarbeit zwischen einem Architekten und dem Service des Phares et Balises (französische Verwaltung für Leuchtfeuer), die sich damals als eine Ausnahme erwies, gab jedoch auch Anlass zu gewissen Auseinandersetzungen. Die Erhöhung des bestehenden Turmes des Oberfeuers und die Errichtung des neuen Unterfeuers führen eine eigenartige Architektur vor Augen, die im Vergleich zu dem traditionellen Leuchtturmbau sowie als Bauwerk in der Baskischen Landschaft überrascht. Diese beiden schlanken Bauten zeichnen sich aus durch das subtile Spiel der stufenförmig angelegten Volumen mit dem völlig nüchternen Dekor, das einfach aus einigen Reliefelementen besteht, die die weißen Fassaden in dem Licht beleben lassen. Pavlovsky, der sich am Anfang seiner Karriere durch einen schlichten Neo-Baskischen Stil auszeichnete, wusste sich hier von dem regionalen Gepräge abzulösen. Er errichtete tatsächlich ein viel moderneres Werk, wie er es gerne ab dem Ende der 20ger Jahre pflegte. Die Leuchtfeuer von Saint-Jean-deLuz sind besonders repräsentativ für die von dem Architekten geschaffene Synthese zwischen ausgesprochener Modernität und Baskischer Tradition, welcher er noch anhing.

\section{AUTEUR}

\section{FABIENNE DOULAT}

Fabienne Doulat a étudié l'architecte Guillaume Abel Blouet (1795-1853) à l'École pratique des hautes études sous la direction de Jean-Michel Leniaud et a écrit plusieurs articles sur le travail de cet artiste : « Guillaume Abel Blouet, du village de Passy aux côtes de Morée, exemplarité ou exception du parcours intellectuel et artistique d'un enfant du peuple », LHA, 2003, nº5, «Guillaume Abel Blouet (1795-1853), architecte de la colonie de Mettray, théoricien et acteur de la réforme pénitentiaire », dans Luc Forlivesi dir., Éduquer et punir. La colonie agricole et pénitentiaire de Mettray (1839-1937), 2005 et « Guillaume-Abel Blouet et le château de Fontainebleau, une approche historique du travail », LHA, n 9, 2005. Elle s'est particulièrement intéressée à la question de l'architecture carcérale et, outre une étude pour le ministère de la Justice, elle est l'auteur de « La prison et son architecture, de la France rurale à celle des grands ensembles ", dans Reconstruire pour moderniser l'institution pénitentiaire, ministère de la Justice ; « La Roche-sur-Yon et Pontivy, prisons du Premier Empire » dans Gilles Bienvenu et 
Géraldine Texier-Rideau dir., Autour de la ville de Napoléon, Rennes, PUR, 2006, ainsi que « La photographie de prison, image d'une réalité architecturale ", dans L'Impossible photographie : prisons parisiennes 1851-2010, Paris, Paris-musées, 2010. Elle a également publié « Le fonds de livres d'architecture à la bibliothèque de l'École des beaux-arts au XIX ${ }^{\mathrm{e}}$ siècle. La bibliothèque et son public ", dans Le livre d'architecture, actes du colloque au Collège de France en novembre 2001. Elle a écrit « L'École des Beaux-arts » dans Les bibliothèques parisiennes, architecture et décor, Paris, AAVP, 2002 et « Les ponts et les quais » dans Autour de Notre-Dame, Paris, AAVP, 2003. Adresse électronique : fabienne.doulat@gmail.com 